Full length article

\title{
Dynamic spectrum access in LTE-advanced networks
}

\author{
Juan D. Deaton ${ }^{\mathrm{a}, \mathrm{b}, *}$, Ryan E. Irwin ${ }^{\mathrm{a}}$, Luiz A. DaSilva ${ }^{\mathrm{a}, \mathrm{c}}$ \\ a Wireless@VT, Bradley Department of Electrical and Computer Engineering, Virginia Tech, Blacksburg, VA, United States \\ ${ }^{\mathrm{b}} \mathrm{NEHS}$ Directorate, Idaho National Lab, Idaho Falls, ID, United States \\ ${ }^{\mathrm{c}}$ CTVR, Trinity College, Dublin, Ireland
}

\section{A R T I C L E I N F O}

\section{Article history:}

Received 5 July 2011

Received in revised form 11 April 2012

Accepted 4 November 2013

Available online $\mathrm{xxxx}$

\section{Keywords:}

Dynamic spectrum access

Long term evolution-advanced

Wireless network architecture

Cognitive radio

\begin{abstract}
A B S T R A C T
As early as 2014, mobile network operators' spectral capacity will be overwhelmed by the demand brought on by new devices and applications. To augment capacity and meet this demand, operators may choose to deploy a Dynamic Spectrum Access (DSA) overlay. The signaling and functionality required by such an overlay have not yet been fully considered in the architecture of the planned Long Term Evolution Advanced (LTE+) networks. This paper presents a Spectrum Accountability framework to be integrated into LTE+ architectures, defining specific element functionality, protocol interfaces, and signaling flow diagrams required to enforce the rights and responsibilities of primary and secondary users. We also quantify, through integer programs, the benefits of using DSA channels to augment capacity under a scenario in which the LTE+ network can opportunistically use TV and GSM spectra. The framework proposed here may serve as a guide in the development of future LTE+ network standards that account for DSA.
\end{abstract}

(C) 2013 Published by Elsevier B.V.

\section{Introduction}

With at least 20 commercial deployments since 2009, Long Term Evolution (LTE) has the fastest adoption rate of any mobile network technology to date. More than 200 operators in 80 countries are investing in LTE, positioning

\footnotetext{
This work was supported by the Idaho National Laboratory (INL) Ph.D. Candidate Program and Virginia Tech Bradley Fellowship. Work supported through the INL is supported through the Laboratory Directed Research \& Development (LDRD) Program under DOE Idaho Operations Office Contract DE-AC07-05ID14517. The United States Government retains and the publisher, by accepting the article for publication, acknowledges that the United States Government retains a nonexclusive, paid-up, irrevocable, worldwide license to publish or reproduce the published form of this manuscript, or allow others to do so, for United States Government purposes. The views and conclusions contained in this document are those of the authors and should not be interpreted as representing the official policies, either expressed or implied, of the Department of Energy or the U.S. Government.

* Corresponding author at: Wireless@VT, Bradley Department of Electrical and Computer Engineering, Virginia Tech, Blacksburg, VA, United States. Tel.: +1 2083514584.

E-mail addresses: juan.deaton@gmail.com, deatjd@vt.edu (J.D. Deaton), rei@vt.edu (R.E. Irwin), ldasilva@vt.edu (L.A. DaSilva).
}

LTE as the future global standard for mobile wireless technologies [1]. Despite the rapid deployment of LTE networks, smart phone devices and applications will create a data tsunami so massive the Federal Communications Commission (FCC) is predicting a $300 \mathrm{MHz}$ spectrum deficit for mobile wireless broadband by 2014 [2]. In a separate report, the FCC also found that the spectrum is mostly underutilized throughout time and space [3]. Therefore, one option for network operators is to increase spectral capacity through a Dynamic Spectrum Access (DSA) overlay, which provides extra capacity through secondary opportunistic spectrum use. One key feature of Long Term Evolution Advanced (LTE+) is the possibility of creating transmission bandwidths, i.e. carriers, of $100 \mathrm{MHz}$ through spectrum aggregation (the aggregation of discontiguous spectrum channels) [4]. Spectrum aggregation brings likely the possibility of hybrid carriers, which consist of a combination of licensed and DSA channels [5].

We agree with the letter from the editors [6] that the number of aggregation scenarios will be limited, especially in the case of DSA. However, we believe that if public policy and regulation on dynamic spectrum access evolves fast enough, LTE+ networks will be able to 
opportunistically use TV white spaces and Global System for Mobile Communications (GSM) spectrum, to augment the licensed use of the spectrum dedicated to LTE+. Both the FCC and the Office of Communications (OFCOM) in the UK have recently issued regulation allowing the opportunistic use of TV white spaces [7,8]. We believe that mobile network operators can take advantage of this new source of spectrum and use it to increase network capacity, and that this additional spectrum may be critical for operators to meet their capacity and coverage demands. Additionally, legacy technologies, such as GSM, still have wide deployments. According to 4G Americas, GSM still has 77\% worldwide penetration and its use will decrease as LTE+ networks increase [9]. Therefore, it would seem cogent to opportunistically use GSM frequencies, while GSM experiences less spectrum occupancy from operators migrating users from GSM to LTE+. Through predicting demand and spectrum vacancies, future LTE+ networks could dynamically self-configure, request, and assign spectrum resources through hybrid carriers.

Although hybrid carriers can provide additional capacity, they will also require modifications to existing architectural models to support the use of DSA carriers. Work in $[10,11]$ proposes a spectrum broker architecture to manage a segment of spectrum known as the Coordinated Access Band (CAB). Through spectrum leases, the spectrum broker issues channels from the $C A B$ for use in cellular networks. The work in [12] proposes a Dynamic Spectrum Access Protocol (DSAP) for coordinating spectrum access among arbitrary wireless technologies on a per-host basis. Works in IEEE 802.22 Wireless Regional Area Network (WRAN) and 802.11af (WhiteFi) [13] are also proposing architectural frameworks for supporting the use of TV white spaces [14] through a combination of sensing techniques and database queries coordinated by a spectrum manager. None of these papers, however, discusses the architectural and signaling elements required to incorporate DSA into $4 \mathrm{G}$ networks such as those using LTE+.

This paper extends our previous work [15] to provide an architectural and operational framework to support a DSA overlay for LTE+. We call this framework Spectrum Accountability (SA). We propose the architectural elements needed to incorporate DSA into LTE+ standards. In our SA framework, an Evolved Node B (eNB) with cognitive capability, called a cognitive Base Station (cBS), issues spectrum lease requests. The cBS sends these lease requests to a centralized entity that manages spectrum lease policies, sets spectrum access rules, and issues leases. At the end of the lease, or at periodic intervals, the cBS reports usage metrics. Regulators or spectrum managers can use these metrics for detecting violations, resolving conflicts, and setting DSA policies. The SA framework provides the means by which regulators can define, enforce, and manage spectrum access rules among competitive secondary operators and protect primary operators from harmful interference.

Our paper makes the following contributions. First, we analyze the effects of a DSA overlay on the operational architecture of LTE+. We propose modifications to existing interfaces and network elements to support the DSA architecture. Our second contribution is our SA framework, which provides the mechanisms to coalesce many different aspects of DSA into specific network element functionality, protocol interfaces, and signaling flow diagrams, in a manner that is agnostic to the spectrum policy paradigm [16]. The SA framework focuses on the operational signaling scenarios to support cooperative sensing techniques [17] and coordination and monitoring of spectrum access through an Spectrum Accountability Server (SAS). In our final contribution, we determine the optimal assignment of LTE+ channels in a hybrid carrier network. We develop and apply integer programs to a scenario in which an LTE+ network uses TV white spaces and also shares spectrum from a GSM system. Through our integer programs we illustrate the quantifiable benefits of using hybrid carriers in an LTE+ network.

Our paper is organized as follows: in Section 2, we briefly outline the LTE+ network elements affected by the DSA overlay, examine the affected LTE+ control planes, and introduce new network elements required to support DSA. Next, in Sections 3 and 4, we describe the operational procedures that comprise the SA framework. In Section 3, we present the operational procedures used to support service requests. Specifically, we describe the following procedures: cBS Registration and Neighbor Discovery, Periodic Cooperative Sensing, Spectrum Request and Reporting, Spectrum Sharing, and Service Request. In Section 4, we present how SA performs spectrum management through the following procedures: New Primary User Alert, Integrated Receiver (IR) Interference Alarm, High Interference Spectrum Lease, Spectrum Unavailable Alarm, and Rogue Transmitter Detection. In Section 5, we use integer programming to model LTE+ networks augmented with a DSA overlay and illustrate the potential benefits. We conclude in Section 6 with a summary of the paper and discuss how our contributions can shape future work in this area.

\section{Effects of a DSA overlay in LTE+}

In this section, we provide an operational and architectural overview of the effects of a DSA overlay in LTE+ networks. This section first presents a brief overview of the LTE+ architecture, followed by an introduction of the proposed DSA network elements. We also suggest how a cBS might deploy and request spectrum from the SAS in the form of a spectrum lease. The section closes by presenting the reference architecture, which illustrates the logical signaling endpoints and introduces a basic scenario for our operational procedures presented in Sections 3 and 4.

\subsection{Affected LTE+ network elements}

Fig. 1 shows our proposed modified LTE+ architecture with new network elements to support a DSA overlay (it also includes an acronym list for convenience). In LTE+, the User Equipment (UE) is the end user, which only has access to packet-switched services, i.e. no circuit switched voice. Through the LTE+ air interface (LTE+$\mathrm{Uu}$ ), the UE connects to the LTE+ network using the eNB, where the eNB has the important function of Radio Resource Control (RRC). RRC has responsibility for the establishment, configuration, maintenance, and release of radio bearers. After establishing radio bearers, the Mobility 


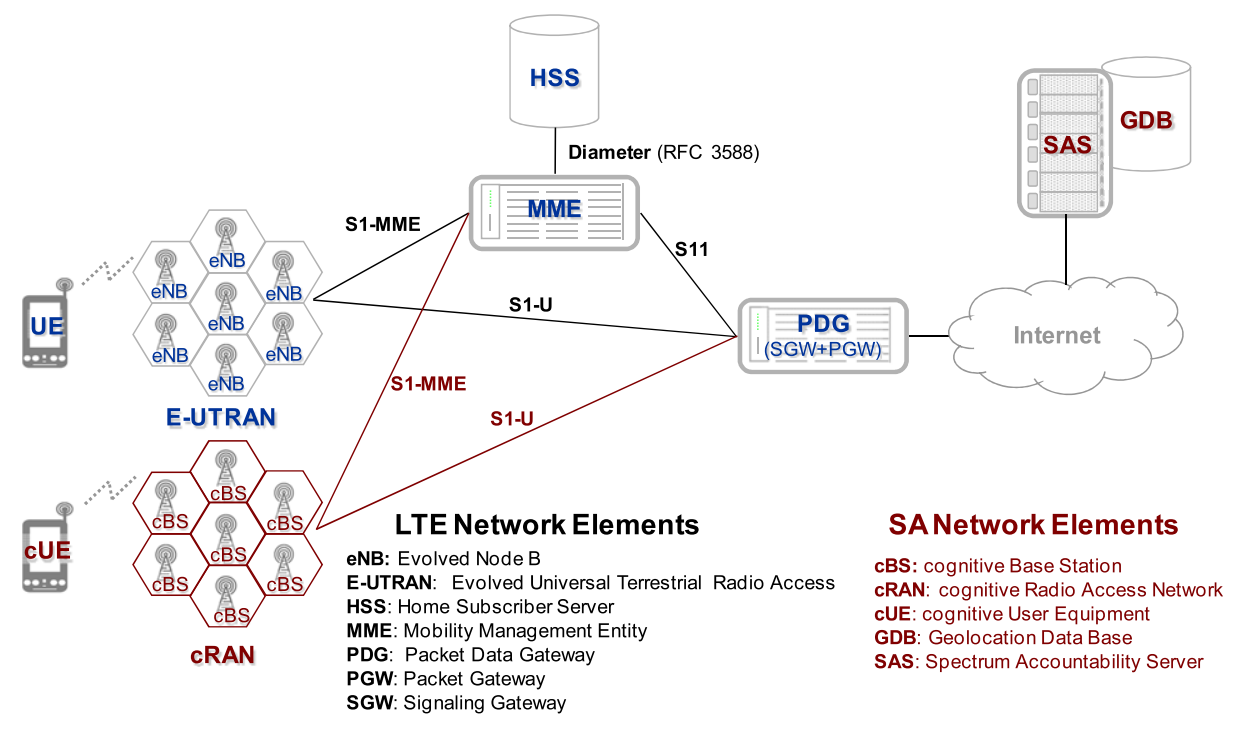

Fig. 1. DSA overlay in an LTE+ architecture is supported through the introduction of the SAS, cBS and the cUE.

Management Entity (MME) and the Home Subscriber Server (HSS) perform Authentication, Authorization and Accounting (AAA) tasks with the UE. The MME uses a signaling protocol called non-access stratum for the UE to register for network services and to support encryption. The HSS houses the access database, similar to a Home Location Register, which contains a record of the UE and the corresponding supported service capabilities. In addition to supporting access and security services, the MME also provides the mechanisms for coordinating data bearers for the UE through the eNB and Packet Data Gateway (PDG). The PDG provides the connections for the UE to external packet networks. (For the ease of discussion, we consider the SGW and PGW LTE+ network elements together as the PDG.) While this brief introduction of LTE+ is sufficient to support further discussion in this study, the reader is encouraged to consult [18] for more detailed information.

\subsection{DSA elements and functions}

We propose to support the DSA overlay through the introduction of the Spectrum Accountability Server (SAS), cognitive Base Station (cBS), and cognitive User Equipment (cUE) shown Fig. 1. The SAS manages spectrum access policies and monitors spectrum leases. Spectrum access policies are sent to the cBS and are then distilled into spectrum access rule sets for spectrum lease requests or opportunistic use of spectrum. ${ }^{1}$ Additionally, the SAS maintains a geolocation database that contains the IP addresses of all cognitive Base Station (cBS) and TV broadcasters, if available. The database also maintains spectrum lease and usage information for spectrum management. In addition to maintaining the database, the

\footnotetext{
1 cBS rule sets could include but are not limited to detection thresholds, power spectrum masks, or maximum power limits for spectrum channels. Work in [19] evaluates and defines some spectrum access rule sets.
}

SAS performs spectrum management through monitoring usage metrics from the cBS, Key Performance Indicators (KPI), and alarms from Integrated Receivers (IRs). Upon detection of harmful interference, the IR, e.g. an IPconnected TV, can send interference alarms to the SAS through the Spectrum Accounting Protocol (SAP). Using SAP, the SAS maintains spectrum-leasing policies, coordinates spectrum leases, monitors spectrum usage, and manages spectrum access rules. The registration and reporting control plane to support SAP is shown in Fig. 2.

In the SA framework, communication among external network entities is required to support cooperative sensing and spectrum trading with geographic neighbors. To support communication with external network entities, the CBS must have an external IP address and a default bearer through the PDG. This functionality does not exist in current LTE+ standards. To establish an external IP address, the cBS should register with the MME, similar to a UE, to create a default data bearer with the PDG for signaling to external network entities. We assume a default bearer for CBS signaling for external network communication. Using external signaling interfaces, the cBS registers with the SAS to find the IP addresses of geographic neighbors, issue spectrum lease requests, and report KPI.

The cUE carries all the same functionality as the UE. However, the cUE also has a spectrum agile radio, capable of operating on and sensing using multiple bands as directed by the network. We call the sensing function and protocol Radio Resource Control-Spectrum Sensing (RRC-SS). The cUE uses a cBS for network service. Like the cUE, the cBS is also capable of spectrum sensing and determining the need for spectrum lease requests. Additionally, the cBS provides the capability of coordinating spectrum sensing with the cUEs and combining the information obtained from the cUEs with its own sensing to produce a spectrum utilization snapshot. This CBS coordinating and combining sensing function is named Radio Resource Control-Cooperative Sensing (RRC-CS). The control planes to support cooperative sensing are shown in Fig. 3. 

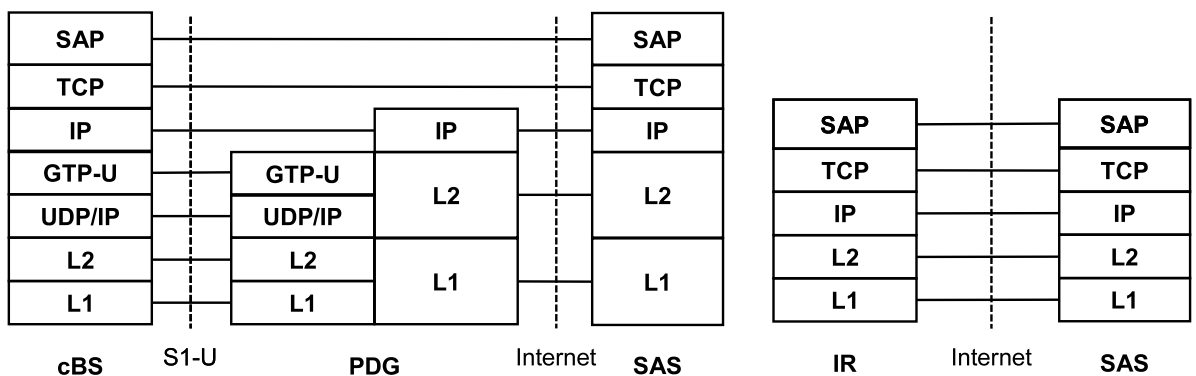

Fig. 2. SAP control plane for cBS registration and reporting functions.

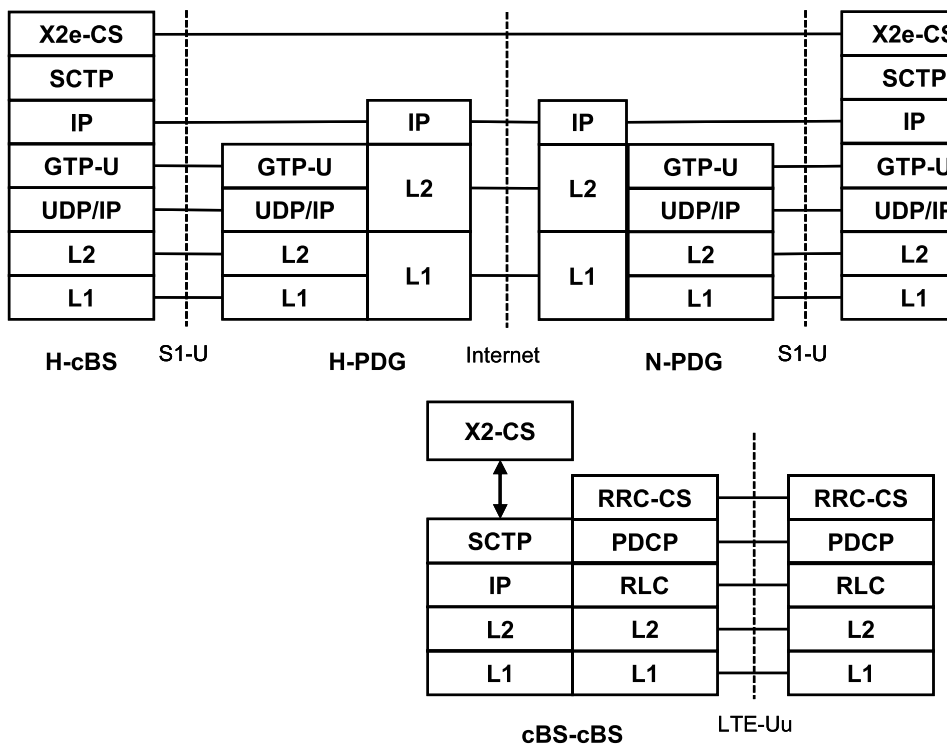

Fig. 3. Cooperative sense control planes for external (top) and internal (bottom) networks. H- and N- indicate home and neighboring networks, respectively.

\subsection{Requesting and deploying DSA carriers}

LTE+ deploys spectrum as carriers on the eNBs to support traffic; e.g., an LTE+ carrier size of $3 \mathrm{MHz}$ consists of 15 resource blocks used for radio bearers. In our study, licensed and DSA sub-carriers comprise a hybrid carrier, where spectrum rights for licensed sub-carriers are through a spectrum license and DSA carriers through a spectrum lease. Since spectrum for DSA sub-carriers could differ by geographic location, DSA sub-carriers should support only data bearers whereas the licensed sub-carriers should support control channels and data bearers. In this way, cUE establishes initial communication with the cBS on a predetermined licensed channel, avoiding the rendezvous problem [20]. If the license spectrum capacity is exceeded, DSA sub-carriers could be used to serve additional traffic. Fig. 4 shows an IDLE cUE requesting a radio bearer on the licensed sub-carrier, i.e. using the Common Control Channel for an RRC connection request. When the cUE transitions from IDLE to CONN, and if there is no capacity on the licensed sub-carrier it will become connected (CONN) to the DSA sub-carrier. In summary, licensed subcarriers are used to bootstrap DSA sub-carriers for tasks like cUE synchronization and access, while DSA carriers are used to increase cBS operating capacity by adding more traffic channels.

To determine when a lease request should be issued, a $\mathrm{cBS}$ must determine when the demand will exceed licensed carrier capacity. A rise in demand can happen for two main reasons: unanticipated events [21] and growth in services [22]. In both cases, the needed spectrum and duration of the spectrum required must be calculated by the network and conveyed to the SAS when sending the spectrum lease request. Work in [10] proposes a batch method, where leases are processed in periodic intervals. Demand predictions could be combined with sensing information and rule sets from the SAS to determine the spectrum bandwidth to be requested and the requested duration of the lease.

\subsection{Reference architecture and interfaces}

In Fig. 5, we provide a reference architecture to introduce our signaling interfaces and operational procedures. In our reference architecture, we consider a simple usecase in which OPERATOR A and OPERATOR B have adjacent sites. OPERATOR A's network is labeled the home $(\mathrm{H})$ network, and OPERATOR B is the neighboring $(\mathrm{N})$ network. Although we show the simple case of a single neighbor, there 


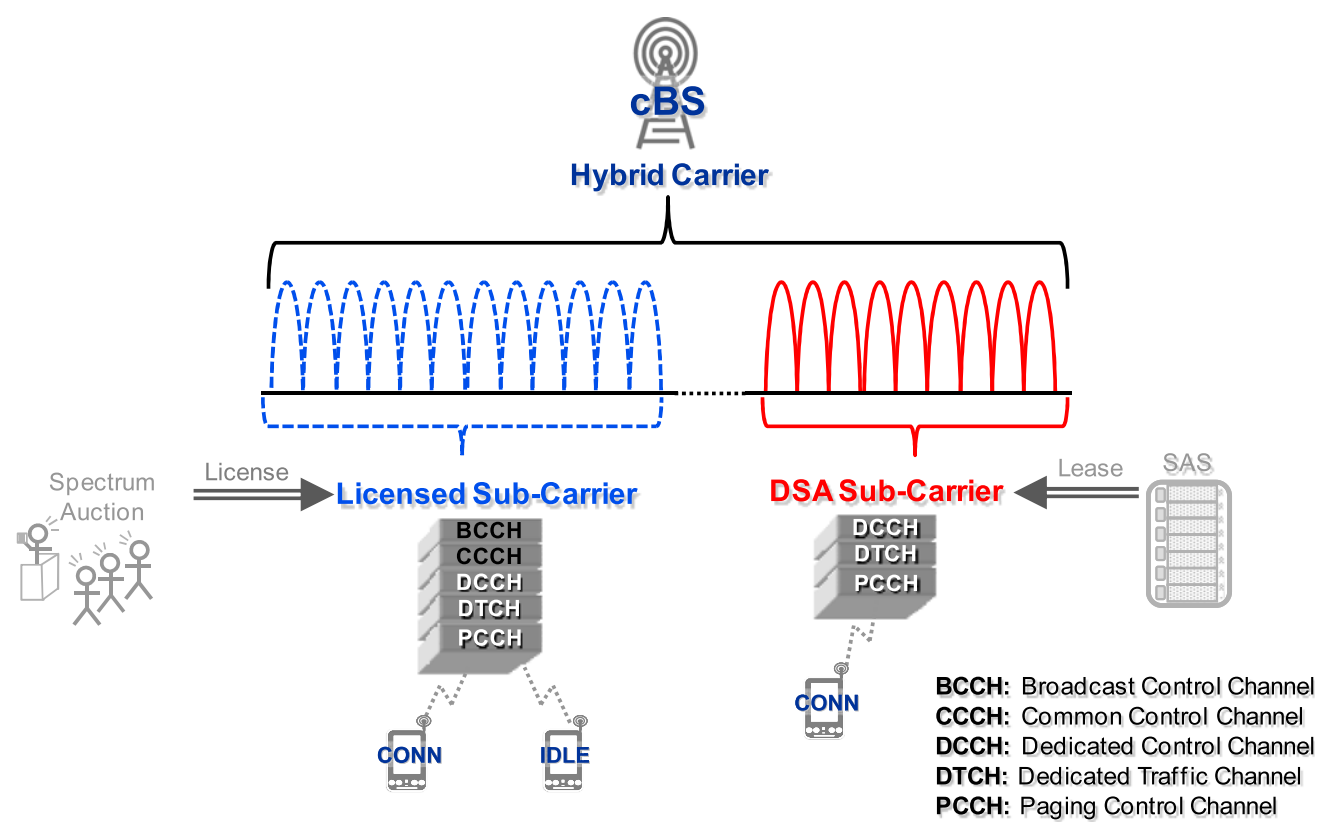

Fig. 4. cBS carrier channel anatomy: the licensed sub-carrier channels shown in black are not supported by the DSA sub-carrier. IDLE mobiles always request access on the licensed sub-carrier. After connected (CONN), cognitive User Equipments (cUEs) use the DSA sub-carrier to support the radio bearer.

could be many. Fig. 5 also captures the protocol and signaling endpoints of SAP, X2e: Cooperative Sense, and X2e: Spectrum Trading. In addition to showing signaling interfaces, Fig. 5 introduces a new network element, the IR.

Fig. 5 also introduces the Spectrum Accounting Protocol (SAP). SAP enables $c B S$ registration, neighbor discovery, KPI reporting for spectrum monitoring, and alarms. Through registration, the Spectrum Accountability Server (SAS) is able to store the locations of geographic neighbors, along with IP addresses, to support neighbor discovery. Using the neighbor discovery information supplied by the SAS, the cBSs form X2 links among themselves to support cooperative sensing and spectrum trading requests. $\mathrm{X} 2 \mathrm{e}$ links are for supporting external network geographic neighbors, whereas X2 links support internal network geographic neighbors. Through SAP, cBSs also report their use of DSA carriers to the SAS via KPI. The KPI contains sets of metrics used to monitor the usage of the DSA carriers. The KPI could track the number of blocked, lost, and successful service attempts, or other metrics, such as block error rates at each cBS. Using KPI, the SAS compiles statistics and provides reports to operators and regulators for monitoring spectrum usage. With IP connectivity, an IR is also able to report loss of service to the SAS using SAP. SAP forms the basis for supporting cooperative sensing, spectrum lease requests, spectrum trading, and spectrum management.

\section{Service request supporting procedures}

Throughout all of our procedure descriptions we remain agnostic of how the SAS decides to allocate leases and when cBSs make lease requests. These decisions could be the result of a specific policy and implementation, whereas our goal is to provide the mechanisms to support a variety of policies. In this section, we first present the operational procedures that support service requests. We use the name service request supporting procedures to emphasize that these procedures are all necessary to establish and support a bearer service to a cUE. We present these procedures in the order that they are likely to occur, similar to "a day in the life" scenario. We have named the operational procedures that support service requests using the DSA overlay as: cBS Registration and Neighbor Discovery, Periodic Cooperative Sensing, Spectrum Lease Request, Spectrum Sharing, and Service Request Procedure.

The cBS Registration and Neighbor Discovery procedure is the genesis of all other procedures. Using SAP, cBS registration is performed to open a spectrum account with the SAS. Registration with the SAS is necessary for validating spectrum lease requests, discovering neighbors, establishing $\mathrm{X} 2 \mathrm{e}$ links with $\mathrm{N}-\mathrm{cBSs}$, and obtaining the spectrum access rules. After registration, the Periodic Cooperative Sensing Procedure could be performed so neighboring cBSs can exchange sensing information to aid in selecting and requesting specific spectrum channels, if required by the spectrum policy. When licensed spectral capacity is exceeded, the Spectrum Lease Request Procedure is triggered to send a spectrum lease request to the SAS, which may leverage sensing information to calculate an interference temperature. In the event that the cBS cannot obtain a lease from the SAS, the Spectrum Trade Procedure can be initiated to obtain a spectrum lease, using licensed or DSA spectrum from a neighboring cBS in similar manner. While trading of licensed spectrum is straightforward, the trade-ability of the DSA spectrum could be specified through spectrum lease from the SAS or spectrum policy. Once the spectrum is obtained, the Service Request Procedure can place overflow traffic onto the DSA carriers. The following subsections provide 


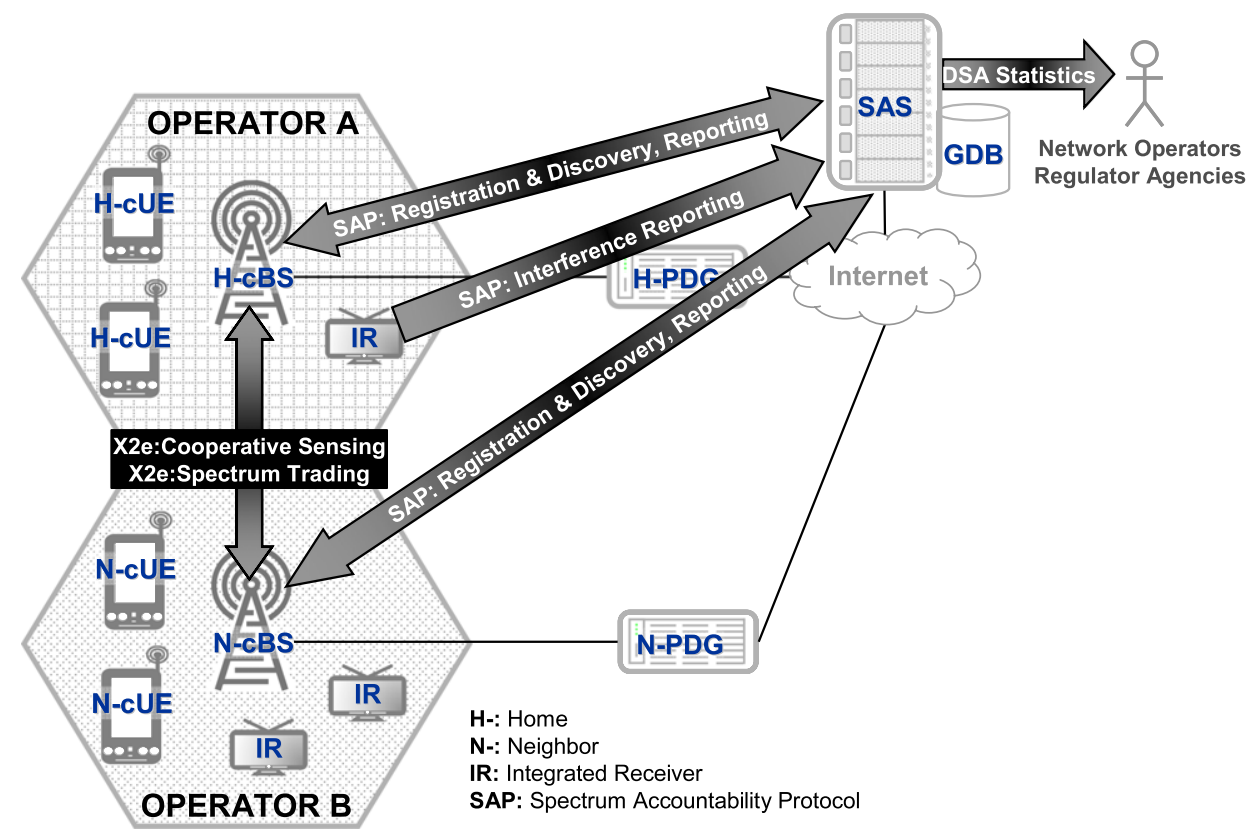

Fig. 5. Reference architecture and logical signaling endpoints.

signaling diagrams for detailed discussion of each of these procedures.

\section{1. cBS Registration and Neighbor Discovery}

The cBS Registration and Neighbor Discovery procedure is shown in Fig. 6. In the first SAP message, the H-cBS sends a registration request to the $S A S$. This registration request contains the IP address of the H-cBS, as well as geolocation information. When this request is received at the SAS, the SAS creates a spectrum account for the H-cBS and updates the geolocation database. The $\mathrm{H}-\mathrm{cBS}$ spectrum account will be used by future N-cBSs to discover the H-cBS and monitor spectrum usage. After the SAS creates the H-cBS account, the SAS responds with a cBS registration response, indicating that the registration was successful, and sends the spectrum access rule set based on the SAS policy. After registration is complete, the $\mathrm{H}-\mathrm{cBS}$ then discovers the $\mathrm{N}$ cBSs through a request/response signaling to the SAS. The SAS neighbor response contains the IP addresses of all the $\mathrm{N}$-cBSs. Using the N-cBS's IP addresses, the H-cBS then sends an X2e: Link setup request to support the exchange of sensing information and spectrum trading with $\mathrm{N}-\mathrm{cBSs}$.

\subsection{Periodic Cooperative Sensing Procedure}

The Periodic Cooperative Sensing Procedure, shown in Fig. 7, supports sensing functions in the Radio Resource Control-Spectrum Sensing (RRC-SS) and the Radio Resource Control-Cooperative Sensing (RRC-CS) protocols. The RRC-SS and the RRC-CS functions are performed by the cUE and the $\mathrm{CBS}$, respectively. The procedure begins with the N-cBS performing spectrum sensing. Subsequently the $\mathrm{N}-\mathrm{cBS}$ issues a cUE Spectrum Sense Order for the cUEs to collect sensing information. Once the cUEs have completed their sensing, this information is sent to the N-cBS. The NcBS then combines the cUE sensing information with its own spectrum sensing and forwards the information to the $\mathrm{H}-\mathrm{cBS}$. The $\mathrm{H}-\mathrm{cBS}$ receives the information and updates the spectrum sensing database. Likewise, the $\mathrm{H}-\mathrm{cBS}$ will provide the sensing information to the N-cBS in the same manner.

\subsection{Spectrum Lease Request Procedure}

At the beginning of the Spectrum Lease Request Procedure, shown in Fig. 8, the cBS has been triggered to send a spectrum lease request because of an increase in traffic. After this trigger, the cBS calculates the parameters of the spectrum lease using the traffic load and spectrum statistics collected from the Periodic Cooperative Sensing Procedure. After determining the spectrum lease parameters, a spectrum lease request is sent to the SAS indicating the desired spectrum channels, bandwidth and period of the request. The SAS examines the request and, using the spectrum-leasing policies, validates the lease and issues the lease to the requesting cBS. After the time period of the lease has expired, the SAS issues a spectrum release order and the $\mathrm{CBS}$ responds with a spectrum release acknowledgment (ACK). In this ACK message, the cBS can also provide the KPI used by the SAS to monitor the spectrum usage. The SAS uses the KPI to update the spectrum account of the $\mathrm{CBS}$, recording how the spectrum lease was used.

In the Spectrum Lease Request Procedure, we have described one simple case. However, different variations of this procedure are also supported. For example, there could be automatic lease renewals or changes to existing lease requests. Automatic lease renewals could be used, for example, to support periodic load from commuter traffic at a roadside cBS, while changes to existing leases would 


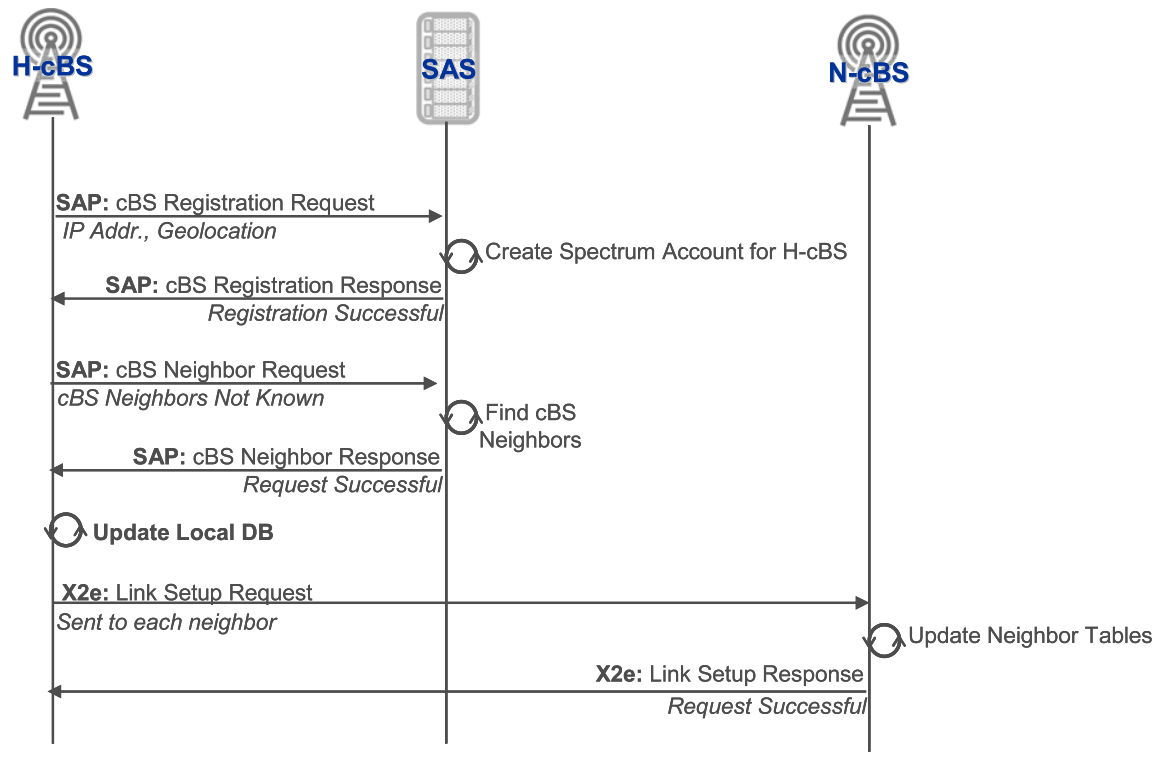

Fig. 6. Registration and neighbor discovery procedure.

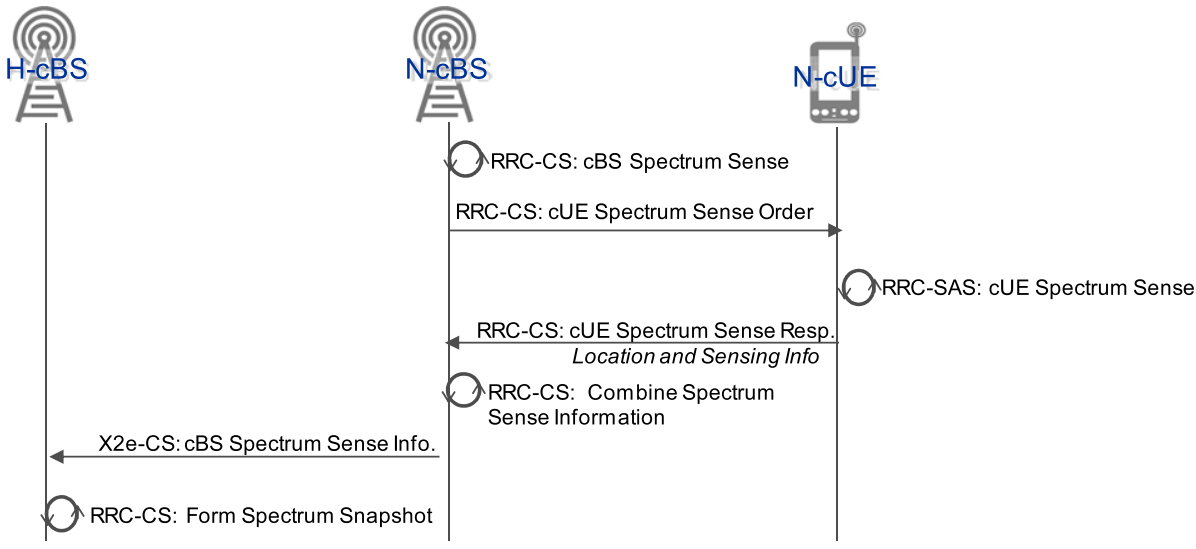

Fig. 7. Periodic cooperative sensing procedure.

account for an increase in overall demand. Additionally, spectrum negotiation could occur as a sub-procedure, where the $\mathrm{cBS}$ and SAS exchange information on spectrum needs and lease availability. Finally, this procedure could be executed on demand or during an off-peak hour when cBS resources are available for performing optimization tasks.

\subsection{Spectrum Sharing Procedure}

As an alternative to obtaining a lease from the SAS, lease requests to the $\mathrm{N}$-cBSs are also a possibility. We call the procedure to make such requests the Spectrum Sharing Procedure (shown in Fig. 9). The Spectrum Sharing Procedure begins with the H-cBS experiencing the same types of triggers as in the spectrum lease request. However, in this case, the SAS cannot validate the lease. As a result, the $\mathrm{H}-\mathrm{cBS}$ inquires with the $\mathrm{N}-\mathrm{cBSs}$ whether there is a lease available, using a Spectrum Lease Request Procedure. The N-cBS examines the request and responds after examining current rules of its valid leases within the H-cBS's geographic area. These rules could either be predetermined by spectrum policy or part of the spectrum lease. The remaining procedure is similar to the Spectrum Lease Request Procedure, with the spectrum release order and ACK being sent to the N-cBS from which the spectrum was obtained.

\subsection{Service Request Procedure}

In the Service Request Procedure, some DSA carrier has been deployed and is in use at the cBS. The procedure, shown in Fig. 10, begins with the cUE issuing a connection request on a licensed carrier. At this point in the procedure, 


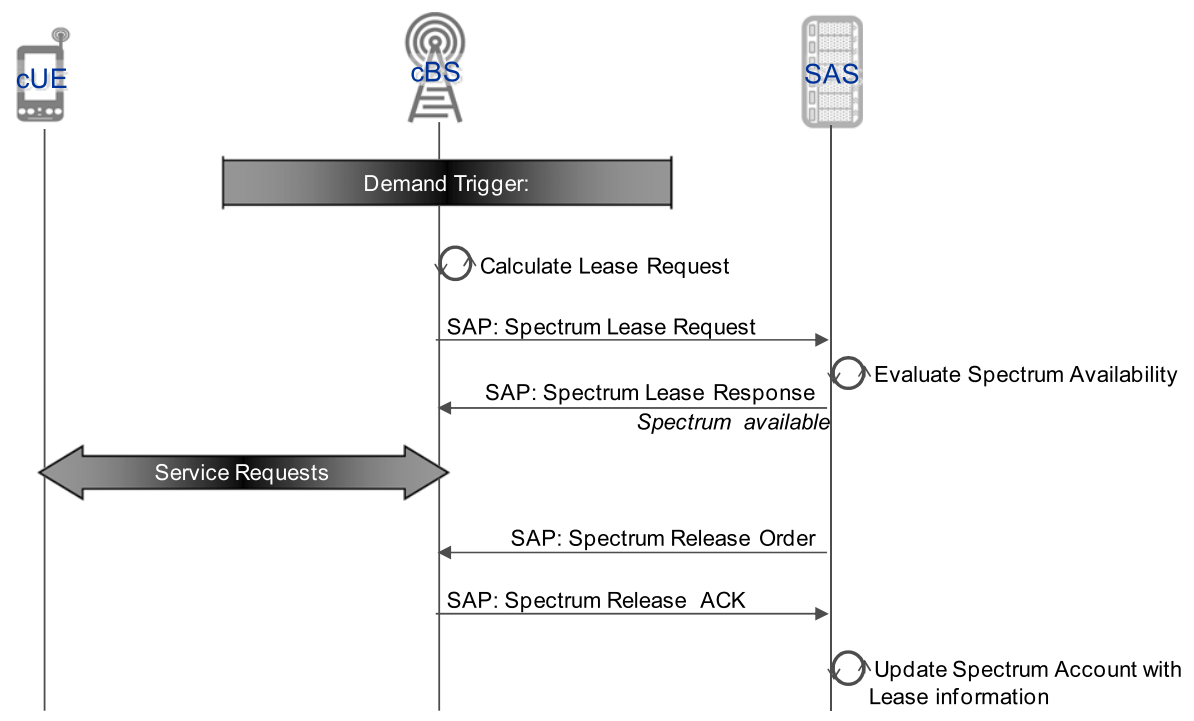

Fig. 8. Spectrum lease request procedure.

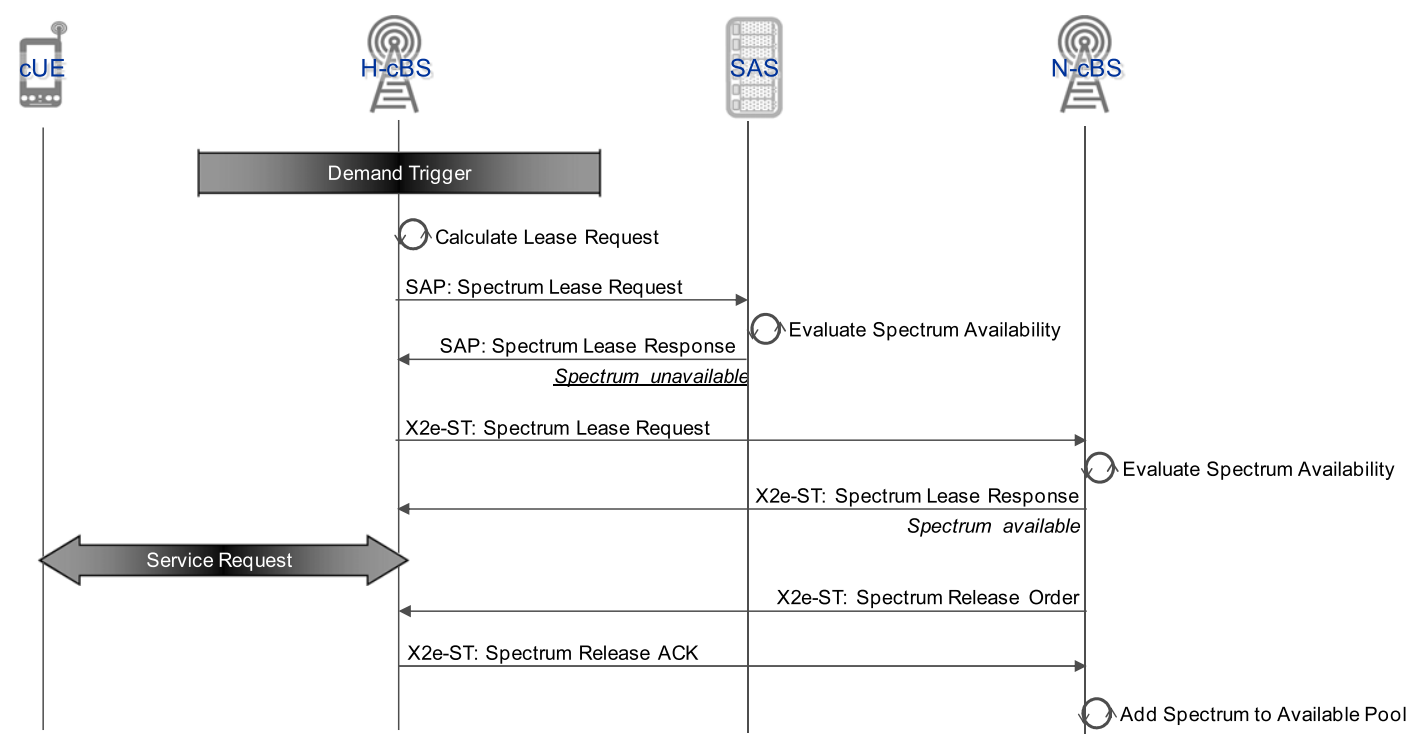

Fig. 9. Spectrum sharing procedure.

the network operator has a policy directing traffic to a specific carrier type. For example, the policy could place all overflow from licensed carriers onto the DSA carrier. In any case, messaging to hand-off CONN_cUEs (connected cUEs) among carriers is considered in this procedure. We highlight this part of the procedure as the spectrum sensing Carrier Optimization sub-procedure. After the cBS has determined which carrier to assign to the IDLE_cUE, a Service Response is sent indicating which carrier the cUE will use. After or during service, KPI are collected from the cUE and the cBS. These KPI are then forwarded to the SAS, which may be done via individual messaging or piggybacked onto the Spectrum Release ACK. We expect incoming handoffs from neighboring cBS would also follow a similar procedure when requesting a connection with the destination $\mathrm{CBS}$.

\section{Spectrum lease management procedures}

In this section, we present operational procedures of the SA framework for spectrum lease management. Spectrum lease management in SA is concerned with monitoring KPI and adjusting spectrum leases to handle problems with interference, performance issues, and policy changes. When changes to spectrum leases are needed, the SAS sends notifications to the affected cBSs, which adjust their local leases and DSA carriers. Additionally, spectrum lease policies could result in changing rule sets of the cBSs. We have identified five new procedures to perform spectrum management: New Primary User Alert, IR Interference Alarm, High Interference Spectrum Lease, Spectrum Unavailable Alarm, and Rogue Transmitter Detection. 


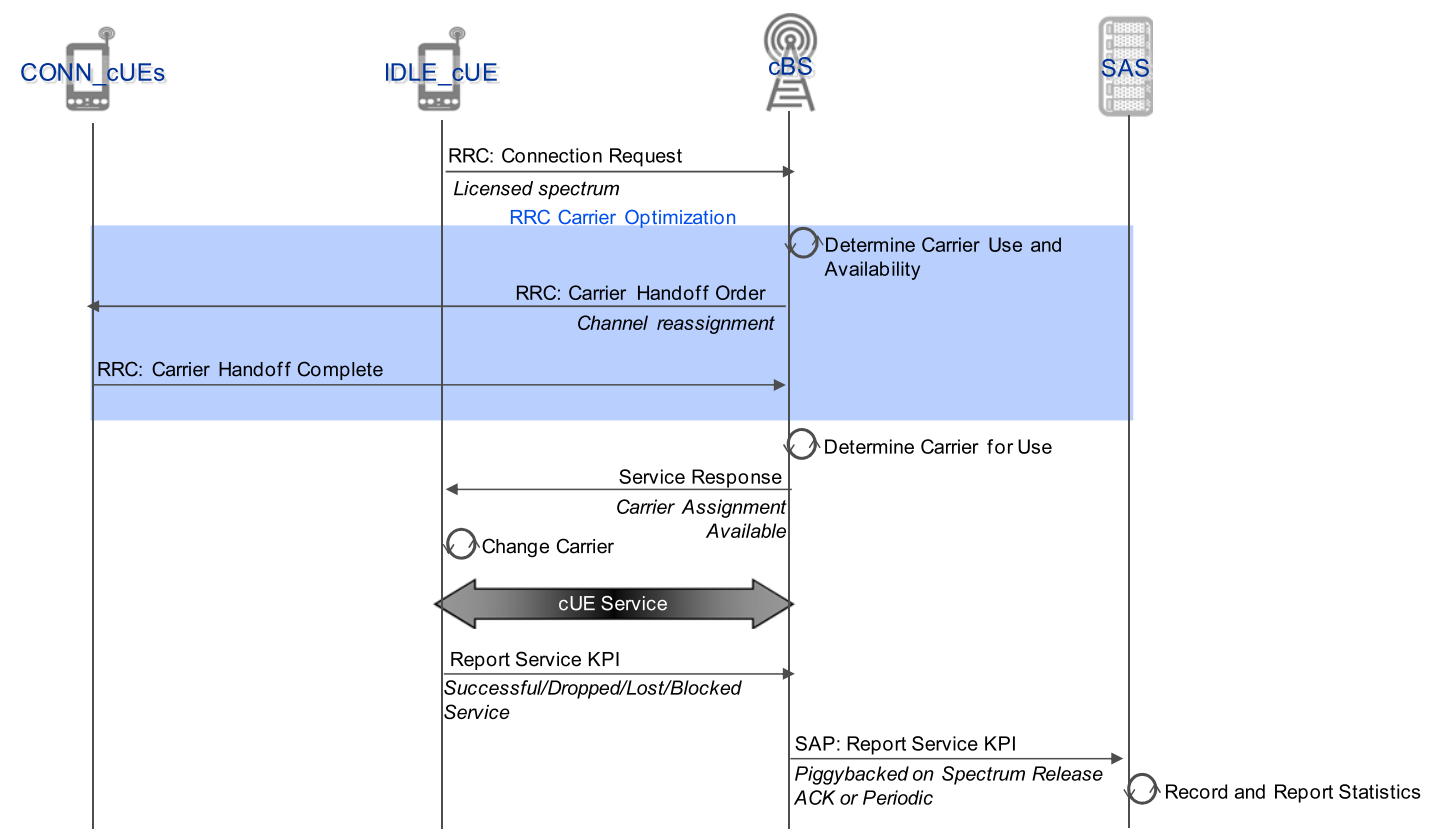

Fig. 10. Service request procedure.

\subsection{New Primary User Alert}

While spectrum sensing is a certainly a valuable tool and method for DSA techniques, it still cannot determine whether hidden receivers are experiencing interference. Additionally, in the event a new primary transmitter becomes present, receivers within the service area may be hidden from secondary transmitters, cBSs, opportunistically using the spectrum. Therefore, the purpose of this procedure is to notify secondary transmitters, cBSs using TV white spaces, of a new primary operator (such as a TV broadcaster), so spectrum can be vacated. The procedure flow diagram is shown in Fig. 11. Using SAP, the primary operator issues a registration request to the SAS. This request contains information about the licensed spectrum, such as center frequency, bandwidth, and licensed geographic area. The SAS updates the geolocation database and returns a registration response. Using the geolocation database, the SAS then identifies and notifies the associated cBSs that there is a new primary operator active on a specific channel. The cBSs then update their spectrum access rules, mark the channel as belonging to a primary operator, and vacate the channel. After the channel is vacated, the $\mathrm{CBS}$ sends an ACK to the SAS. Once all the cBSs have vacated the spectrum, the SAS notifies the primary operator.

\subsection{IR Interference Alarm}

Hidden receivers can be a problem in DSA. In the hidden receiver problem, a primary operator transmits to a primary receiver and a secondary operator, unaware of the primary receiver and the primary operator, interferes. The IR Interference Alarm provides a method to avoid interference to hidden receivers by using the Integrated Receiver (IR) to detect the loss of service and report this loss to the SAS. In this scenario, the IR has knowledge of its location by either a postal address provided by the end user or geolocation provided by GPS. Additionally, the IR is able to discover the SAS by a query to a server, similar to a DNS server, which resolves the proper regional SAS. The procedure, shown in Fig. 12, begins with the IR detecting service loss because of interference. Once this problem has been detected, the IR sends a Service Loss Alarm to the SAS. After receiving the service loss alarm, the SAS analyzes the existing spectrum leases to determine the potential interferers and sends alarms to those cBSs. The cBSs then take some action, such as relinquishing the channel or reducing power.

\subsection{High Interference Spectrum Lease}

The purpose of the High Interference Spectrum Lease Procedure is to detect the spectrum leases that experience high amounts of interference and adjust spectrum policy, if possible. The procedure, shown in Fig. 13, begins with an $\mathrm{H}$-cBS reporting KPIs that indicate poor service or the inability to provide service using the spectrum lease. The SAS analyzes these statistics and makes changes to the set of spectrum leases. It then sends updates to all cBSs that are affected by these changes, and the cBSs update their spectrum leases and carriers accordingly. In addition to adjusting spectrum access rules through a policy change, the SAS can also look for rogue transmitters.

\subsection{Rogue Transmitter Alarm Procedure}

Another possible cause of high call blocking or service loss is a rogue transmitter. A rogue transmitter is a transmitter that uses frequencies without a lease or license. In this procedure, shown in Fig. 14, instead of adjusting the set of spectrum leases the SAS determines the 


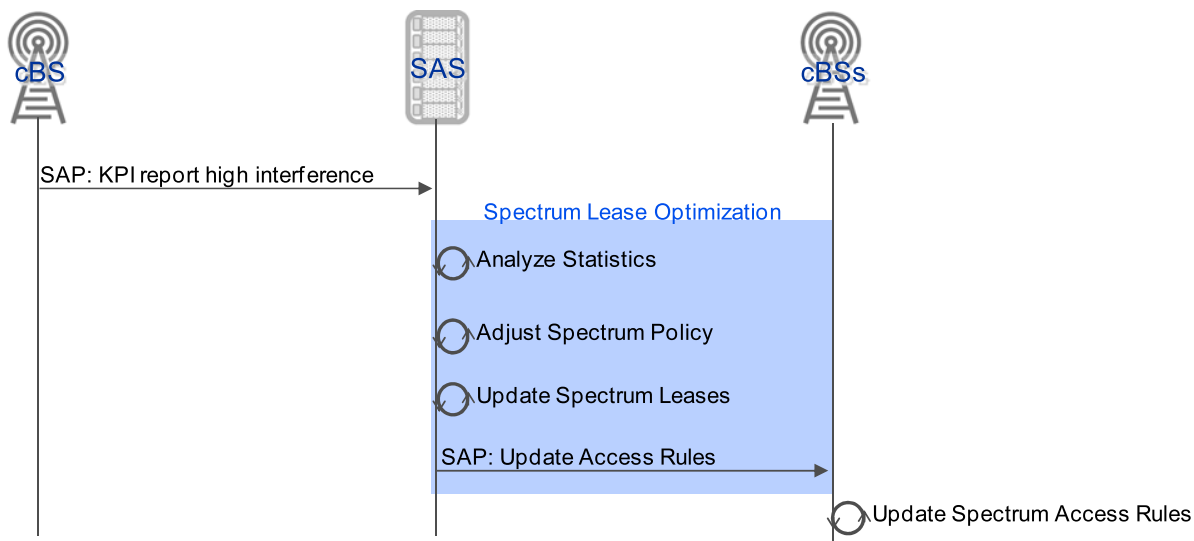

Fig. 11. New primary user alert procedure.

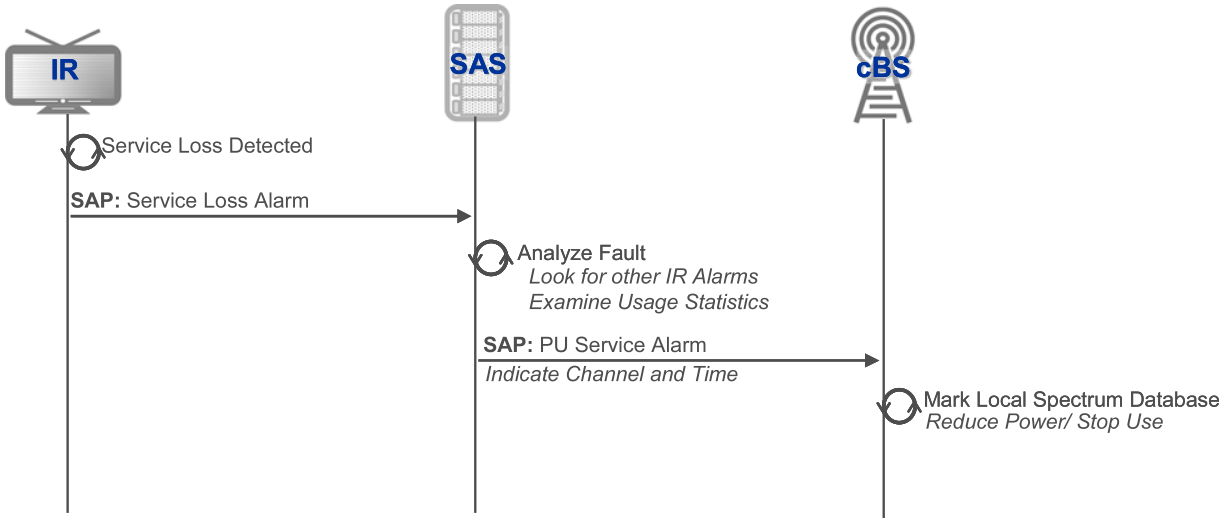

Fig. 12. IR interference alarm procedure.

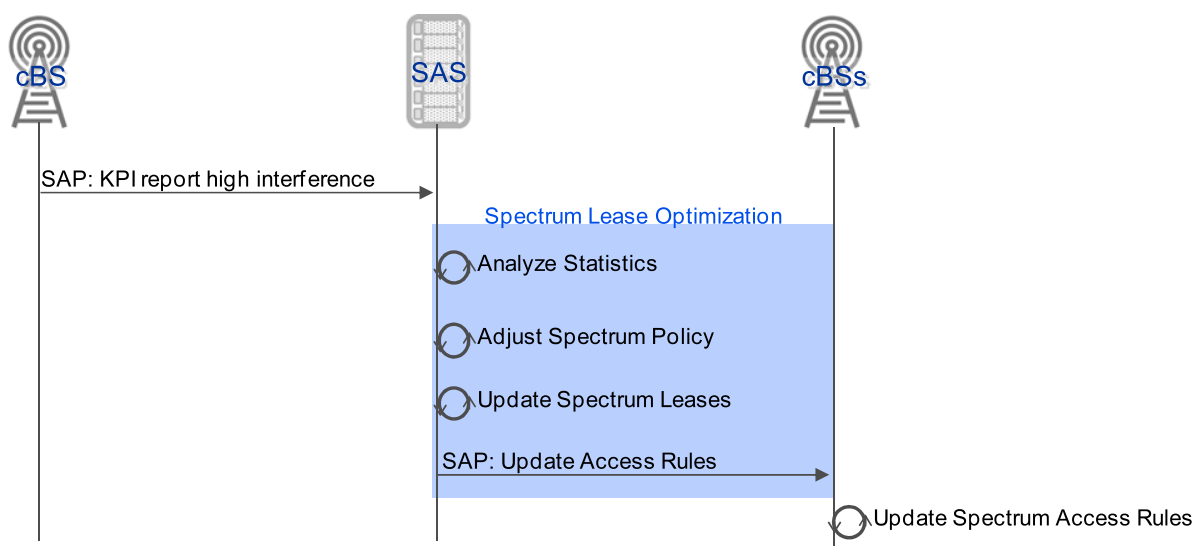

Fig. 13. High interference spectrum lease procedure.

location of the rogue transmitter. After receiving indication that a spectrum lease is experiencing poor service, the SAS determines which cBSs are in the area from which the transmission is occurring. It then sends a Spectrum Snapshot Request to each of the cBSs in the area. Once each cBS replies with the Spectrum Snapshot ACK, the SAS then uses the spectrum sensing information to determine the geolocation of the rogue transmitter. This information is then used by regulators to issue fines or take other appropriate measures.

\subsection{Spectrum Unavailable Alarm}

In this final procedure, a cBS has detected that future demand from customers will exceed its capacity. However, the $\mathrm{cBS}$ is unable to issue a spectrum lease request given 


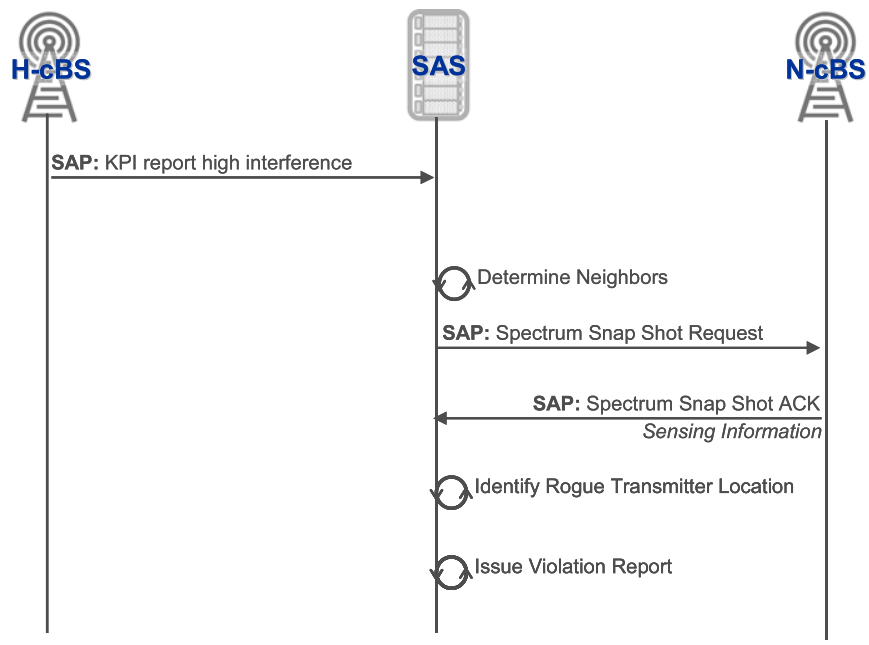

Fig. 14. Rogue transmitter alarm procedure.

the existing rule set from the SAS. This procedure is useful since the SAS is able to notify regulators and operators of either policies that may be overly strict or the simple lack of spectral resources. In this case, the SAS can gradually relax policy restrictions and observe interference alarms from IRs or other cBS. The procedure is shown in Fig. 15. In the first message of the procedure, the cBS identifies a need for more spectrum and begins to calculate a spectrum lease request. When examining the spectrum information and existing rule set, the cBS finds that there is no available spectrum. As a result, the cBS issues a Spectrum Unavailable Alarm to the SAS. In response to the alarm, the SAS examines the current statistics and policy and determines it can change the spectrum leases or adjust the $\mathrm{cBS}$ rule set. The $\mathrm{cBSs}$ then use the updated rule sets to update their future leases.

\section{Optimal assignment of DSA carriers}

In the previous section, we presented the affected control planes, new network elements, operational procedures, and the SA framework to support a DSA overlay in an LTE+ network. In this section, we develop two integer programs considering channel assignment and spectrum aggregation in LTE+ networks. In the first program, we provide a formulation for the basic channel assignment problem in LTE+ networks. In the second program, we consider the aggregation of licensed channels with DSA spectrum through the use of our SA framework. In this scenario, the network is allowed to use TV spectrum, when sufficiently far away from transmitting stations. Additionally, we also assume a GSM overlay with the LTE+ network, owned by the same operator. The LTE+ network with the SA framework senses vacancies in the GSM spectrum and is allowed to use the GSM spectrum if it is unoccupied. Our integer programs represent a snapshot in time, which could be used by LTE+ networks to optimally self-configure during periodic intervals.

In the scenario of interest, an LTE+ network is allowed to assign channels (licensed, DSA, or both) onto cBS carriers to serve a given load [4]. ${ }^{2}$ To manage inter-cell interference in LTE+, channels are aggregated into a cell-edge or cellcenter carrier [23], where channels in the cell-edge carriers must be different among neighboring base stations. An example of this type of frequency assignment is shown in Fig. 16. Our first integer program examines the basic LTE+ channel assignment problem, which considers only demand and assignment constraints given a set of licensed channels. In our second problem, we consider the same scenario with a set of licensed LTE+ channels, a set of TV channels, and set of GSM channels, which can be used to meet the demand.

\subsection{Problem formulation}

In the basic LTE + channel assignment problem, cell edge frequencies must be different between neighboring cells to prevent inter-cell interference. Clearly, channels deployed within the same cell cannot be used by both the cell-edge and cell-center carriers. Licensed spectrum is limited to $W$ channels, which are available to deploy throughout a network with $B$ base stations. Let $x_{i, c, k}=1$ when cBS $i$ assigns channel $c$ to carrier $k$, where $k=1$ and $k=2$ indicate a cell-center and a cell-edge carrier, respectively. Denote $d_{i, k}$ as the demand, in number of channels, at cBS $i$ on carrier $k$. Through an adjacency matrix $\mathbf{N}$, we define neighbor relationships, where $n_{i, j}=1$ indicates that cBS $i$ is a neighbor to cBS $j$. The objective of the network is to maximize the assignment of the channels on each carrier such that the demand at each cBS and carrier is met. Here we present the basic channel assignment problem for an LTE+ network as integer program $\mathcal{P}_{1}$ :

Maximize:

$$
\sum_{i=1}^{B} \sum_{c=1}^{W} \sum_{k=1}^{2} x_{i, c, k} .
$$

\footnotetext{
2 In LTE+, a resource block is based on $180 \mathrm{kHz}$. Without loss of generality, we consider a $200 \mathrm{kHz}$ block for the LTE+ channel.
} 


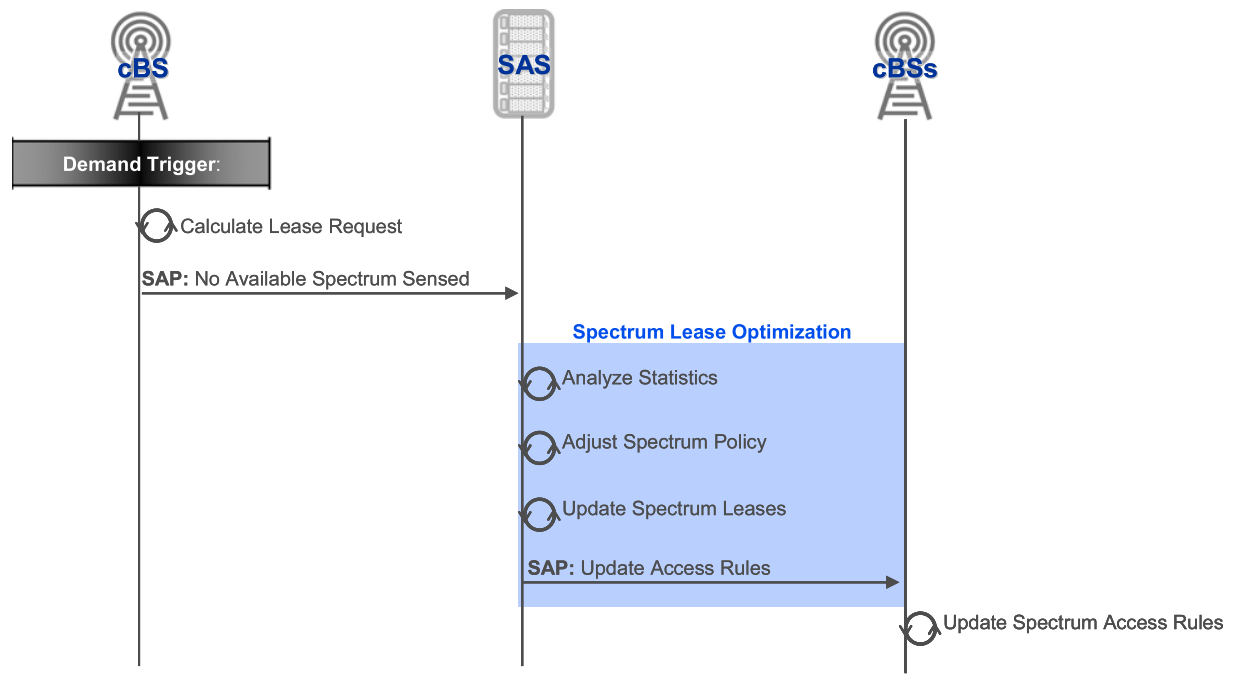

Fig. 15. Spectrum unavailable alarm procedure.

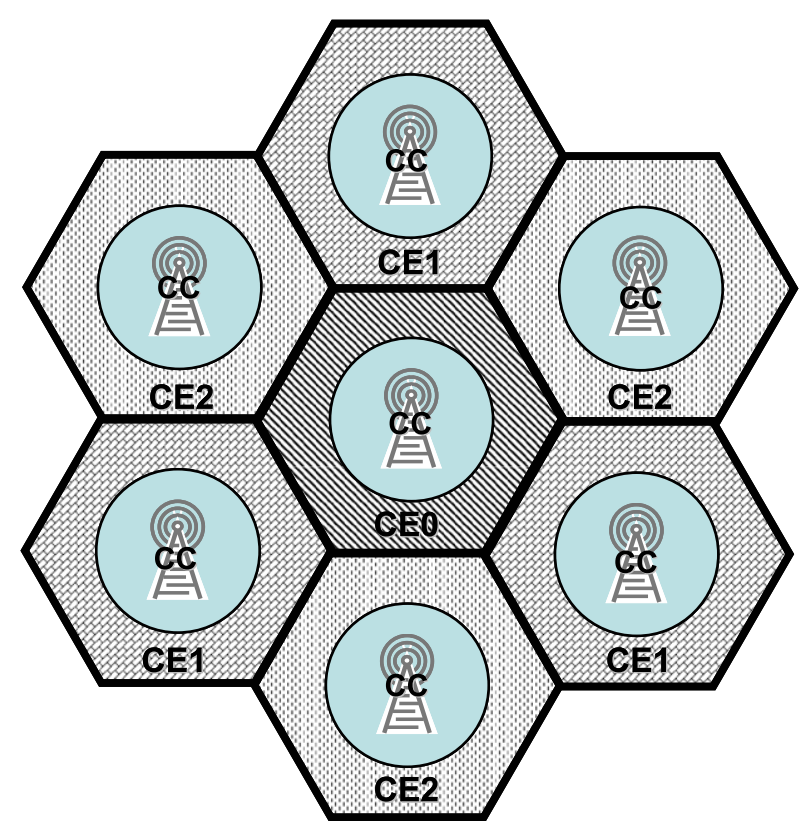

Fig. 16. Example of frequency reuse scheme for LTE+. Cell-center carriers do not generate inter-cell interference, while cell-edge carriers do and are required to be different for neighboring cells.

\section{Subject to:}

$\sum_{c=1}^{W} x_{i, c, k} \leq d_{i, k} \quad \forall i, k$

$\sum_{k=1}^{2} x_{i, c, k} \leq 1 \quad \forall i, c$

$n_{i, j}\left(x_{i, c, 2}+x_{j, c, 2}\right) \leq 1 \quad \forall i, j, c$

$\sum_{c=1}^{W} \sum_{k=1}^{2} x_{i, c, k} \leq W \quad \forall i$

$x_{i, c, k} \in\{0,1\} \quad \forall i, c, k$.
Constraint (2) requires that channels only be assigned as required to meet demand. Constraint (3) only allows channels to be assigned once per cell, i.e. cell-edge and cell center carriers cannot have the same channel. Constraint (4) prevents neighboring cells from having the same channels in the cell-edge carriers. Lastly, Constraint (5) enforces the total channel limit for the network operator.

Leveraging the architecture we proposed in Section 2, cBSs are allowed to reuse TV spectrum and GSM spectrum when available. Channel assignment or assignment rules can be provided by an SAS and knowledge of primary users can be stored in the Geolocation DataBase (GDB). DSA channels can only be used when cBSs will not interfere with primary stations. Therefore, we define an interference 
matrix $\mathbf{P}$, where $p_{i, l}=1$ if $\mathrm{cBS} i$ is not within interfering distance of TV station $l$. We further denote $y_{i, l, c, k}=1$ to indicate when cBS $i$ uses TV station l's channel $c$ on carrier $k$, where there are $P$ TV stations, each with $V$ channels available. GSM channels available at the local cell can also be used when unoccupied. Thus, we define matrix $\mathbf{A}$, where $a_{i, c}=1$ if the base station $i$ 's channel $c$, from the GSM spectrum, is available for secondary use. We further denote $z_{i, c, k}=1$ to indicate when cBS $i$ uses channel $c$, from the GSM spectrum, on carrier $k$, where each base station has $U$ potential channels available. Since GSM frequencies are previously deployed, in our formulation we assume that inter-cell interference coordination is not necessary for their opportunistic use. Therefore, GSM channels can only be opportunistically used within the same cell. We now extend the objective function and constraints from the previous problem to include these new concepts as a new integer program to solve the DSA channel assignment problem $\mathcal{P}_{2}$ :

Maximize:

$$
\begin{aligned}
& \sum_{i=1}^{B} \sum_{c=1}^{W} \sum_{k=1}^{2} x_{i, c, k}+\sum_{i=1}^{B} \sum_{l=1}^{P} \sum_{c=1}^{V} \sum_{k=1}^{2} y_{i, l, c, k} \\
& \quad+\sum_{i=1}^{B} \sum_{c=1}^{U} \sum_{k=1}^{2} z_{i, c, k} .
\end{aligned}
$$

Subject to:

$\sum_{c=1}^{W} x_{i, c, k}+\sum_{c=1}^{V} \sum_{l=1}^{P} y_{i, l, c, k}+\sum_{c=1}^{U} z_{i, c, k} \leq d_{i, k} \quad \forall i, k$

$\sum_{c=1}^{W} x_{i, c, k} \geq 1 \quad \forall i, k$

$\sum_{k=1}^{2} x_{i, c, k} \leq 1 \quad \forall i, c$

$\sum_{k=1}^{2} y_{i, l, c, k} \leq p_{i, l} \quad \forall i, l, c$

$\sum_{k=1}^{2} z_{i, c, k} \leq a_{i, c} \quad \forall i, c$

$n_{i, j}\left(x_{i, c, 2}+x_{j, c, 2}\right) \leq 1 \quad \forall i, j, c$

$n_{i, j}\left(y_{i, l, c, 2}+y_{j, l, c, 2}\right) \leq 1 \quad \forall i, j, l, c$

$\sum_{c=1}^{W} \sum_{k=1}^{2} x_{i, c, k} \leq W \quad \forall i$

$\sum_{l=1}^{P} \sum_{c=1}^{V} \sum_{k=1}^{2} y_{i, l, c, k} \leq \sum_{l=1}^{P} p_{i, l} V \quad \forall i$

$\sum_{c=1}^{U} \sum_{k=1}^{2} z_{i, c, k} \leq U \quad \forall i$

$x_{i, c, k}, y_{i, l, c, k}, z_{i, c, k} \in\{0,1\} \quad \forall i, l, c, k$.
Constraint (8) is analogous to Constraint (2) in $\mathcal{P}_{1}$. However, in the case of the second problem we must also include the DSA channels from TV stations and GSM spectrum. Constraint (9) insures that there is at least one licensed channel per carrier, which is necessary for initial network access as described in Fig. 4. Constraints (10)-(12), analogous to Constraint (3) in $\mathcal{P}_{1}$, prevent a given channel from being used both in the cell-center and the cell-edge-this holds for licensed LTE+ channels as well as TV or GSM channels occupied opportunistically by an LTE+ operator. Additionally, Constraints (11) and (12) ensure that DSA channels are only allocated by the CBS when transmission will not interfere with the primary user. Constraints (13) and (14), analogous to Constraint (4) in $\mathcal{P}_{1}$, prevent neighboring cells from allocating the same channels in the cell-edge carriers. Constraints (15)-(17) limit the number of channels deployed at a cBS to the total number of channels used from the licensed LTE+, TV, and GSM channels sets.

\subsection{Simulated scenarios and results}

To illustrate the use of this formulation for optimal deployment of carriers in an LTE+ network, we use random network configurations as inputs for our integer program and use the CPLEX [24] solver to determine optimal solutions. We do this to show the quantifiable benefits of using DSA spectrum in an LTE+ network. Our scenarios are based on an $8 \times 8$ hexagonal cell lattice, where a cBS, GSM base station, or TV station may reside in the center of a cell. cBSs and GSM base stations reside in all cells and the locations of TV stations are determined by a randomly selected cell. Additionally, TV stations have an interference radius of 2, where any cell outside of the interference radius is allowed to reuse the TV station's spectrum. ${ }^{3}$ This lattice and the primary interference region are shown in Fig. 17.

For our input variables we vary $d_{i, k}, W, V, U$, and the number of available TV stations to share spectrum with. The demand, $d_{i, k}$, is modeled as a uniformlydistributed random variable. We consider scenarios where the network has $W=75$ and $W=150$ LTE+ channels $(15,30 \mathrm{MHz})$ available for distribution among the cBSs. Each TV station has $V=30$ potential DSA channels (6 $\mathrm{MHz}$ ) that can be used by the LTE+ network, when outside the interference radius of the TV stations. Our scenarios consider cases when there is one or two primary TV stations whose channels can be used opportunistically. For GSM spectrum, we consider cases of $U=5$ and $U=10$ DSA channels $(1,2 \mathrm{MHz})$ for each cell, where each DSA channel can be allocated to LTE+ traffic in the same cell if available. The GSM spectrum availability (in particular, the duty cycle of the channel use) is modeled by the modified

\footnotetext{
3 Real TV interference regions have been defined in regulation from the FCC [25] and OFCOM [8], which may be much larger than our interference radius of two cells. In any case, white space availability may differ within a region of this size, which we illustrate through our hypothetical topology.
} 


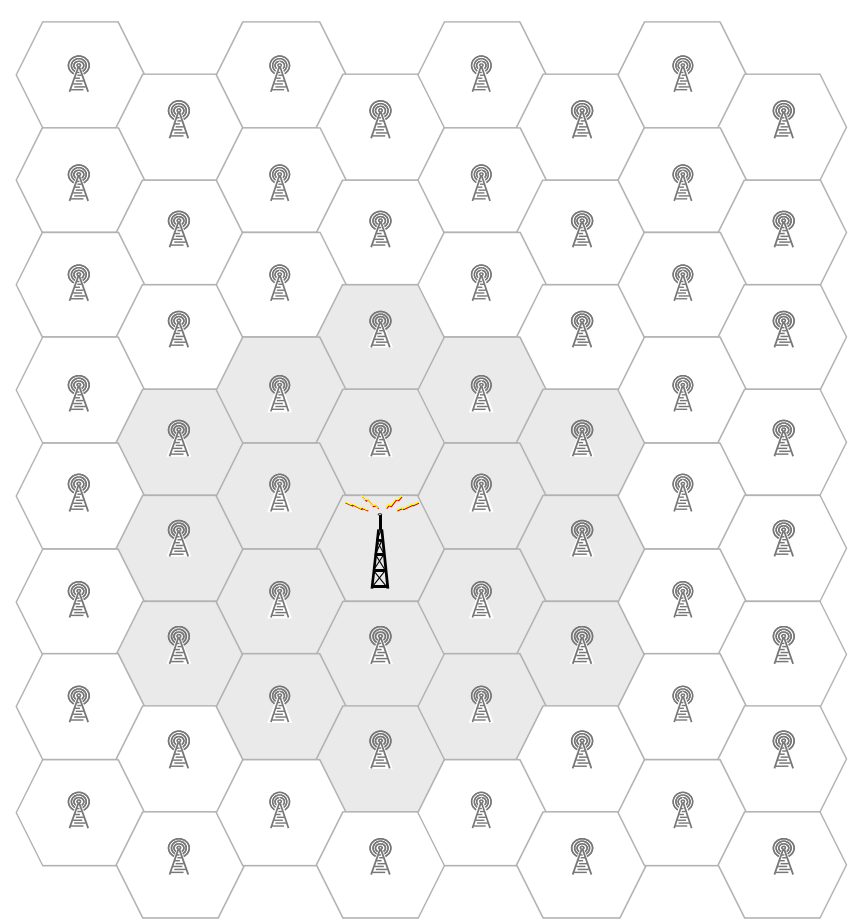

Fig. 17. Simulated hexagonal lattice used to create integer program input parameters. Primary station and interference region are shown in bold and darker cells.

beta distribution, as proposed in [26]. The modified beta distribution probability density function is given by

$$
\begin{aligned}
f_{m \beta}(x ; \alpha, \beta)= & p_{D C=0} \cdot \delta(x) \\
& +\left(1-p_{D C=0}-p_{D C=1}\right) \cdot f_{b}(x ; \alpha, \beta) \\
& +p_{D C=1} \cdot \delta(x-1),
\end{aligned}
$$

where $x \in[0,1], p_{D C=0}$ and $p_{D C=1}$ are parameters used to characterize the duty cycle, $\delta(x)$ is the Dirac delta-function and $f_{b}(x ; \alpha, \beta)$ is the probability density function for the beta distribution, given by

$f_{\beta}(x ; \alpha, \beta)=\frac{1}{\mathscr{B}} x^{\alpha-1}(1-x)^{\beta-1}$,

and where $\mathscr{B}$, the beta function, is given by

$\mathcal{B}(\alpha, \beta)=\int_{0}^{1} t^{\alpha-1}(-t)^{\beta-1} d t$.

The beta function is parameterized by $\alpha$ and $\beta$. Wellens, in [26], developed this model by using the energy detection of $200 \mathrm{kHz}$-wide channels in one-second intervals. Bands were considered available if the measured energy on the channel was below $-107 \mathrm{dBm}$. In our scenario, opportunistic LTE+ channels in GSM spectrum is considered under the same circumstances. We simulate different loading on each base station by randomly selecting one of the parameter sets from Table 1, which represent the distribution of the GSM channels for each base station.

Fig. 18 shows the results of the integer programs using the given scenarios and a baseline of $15 \mathrm{MHz}$ of licensed LTE+ channels. Intuitively, more traffic can be served if the
Table 1

Band parameters used to determine GSM channel duty cycle from [26].

\begin{tabular}{lllll}
\hline Band descriptor & $p_{D C=0}$ & $p_{D C=1}$ & $\alpha$ & $\beta$ \\
\hline GSM900 DL AB & 0.000 & 0.934 & 3.677 & 1.336 \\
GSM900 DL IN & 0.044 & 0.401 & 0.575 & 1.528 \\
GSM1800 DL AB & 0.193 & 0.616 & 0.716 & 1.202 \\
GSM1800 DL IN & 0.344 & 0.111 & 0.698 & 1.317 \\
\hline
\end{tabular}

DSA channel availability is higher. There is a significantly higher increase in total capacity with additional $6 \mathrm{MHz}$ TV channels in comparison to the use of GSM spectrum. GSM spectrum contributes less because in some cases many of the GSM channels are being occupied by primary users, in accordance with [26]. The TV spectrum is always available when cells reside outside the interference radius and thus provides more benefit. As highlighted in our introduction, we would expect the benefits from GSM spectrum to add more benefits as more users migrate from GSM to LTE+. One other observation is that past a certain threshold the curves become almost linear, creating a proportional benefit at a fixed demand. Using this observation, we compare cross sections of fixed demands to show the quantitative benefits of additional DSA spectrum.

Fig. 19 shows the comparison of the additional proportional traffic that can be served given additional DSA spectrum, for a constant demand. The set of bars on the left illustrate the benefits of additional DSA channels under the $15 \mathrm{MHz}$ baseline, while the bars on the right illustrate the $30 \mathrm{MHz}$ baseline. In both cases, we observe that as the amount of TV spectrum increases, the proportion of additional traffic served by GSM channels decreases. Additionally, Fig. 19 shows that with $6 \mathrm{MHz}$ of spectrum 


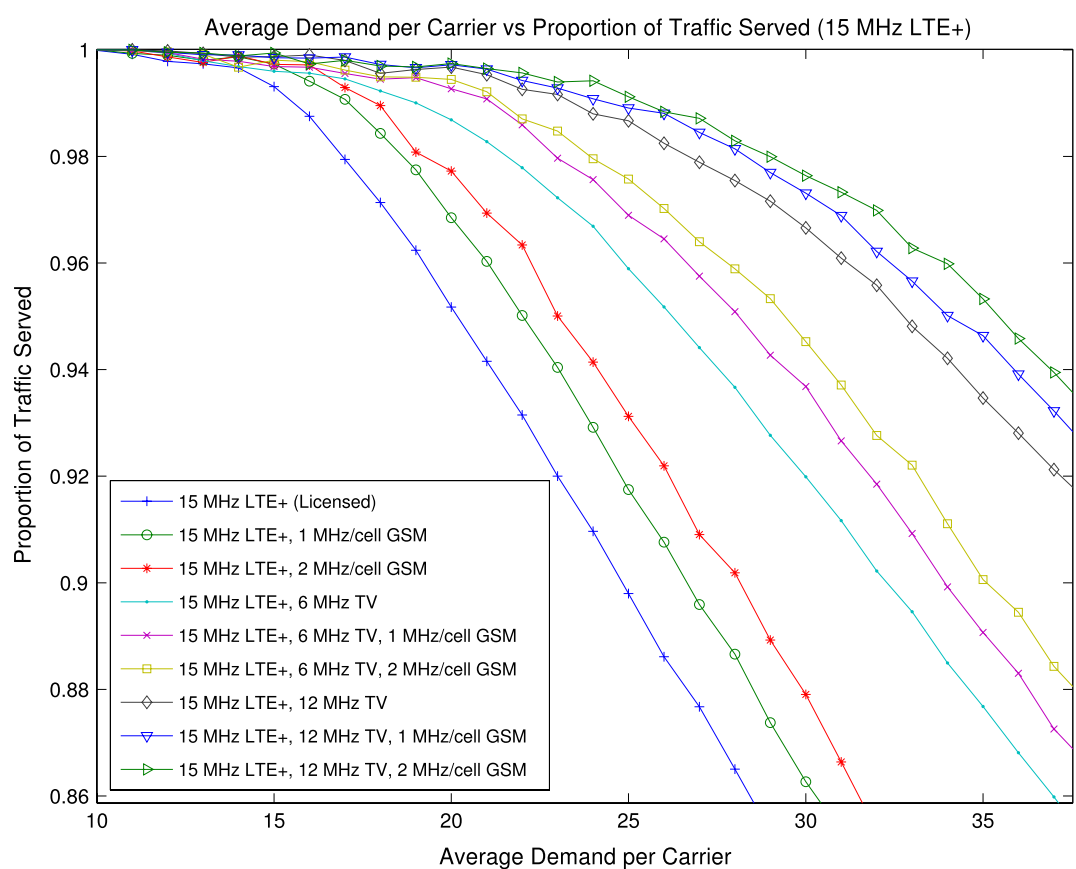

Fig. 18. Optimal channel assignment of $15 \mathrm{MHz}$ of licensed LTE+ spectrum channels compared with the same assignment with additional DSA spectrum. There is a significantly higher increase in overall capacity with additional $6 \mathrm{MHz}$ TV channels in comparison to the opportunistic use of GSM spectrum.

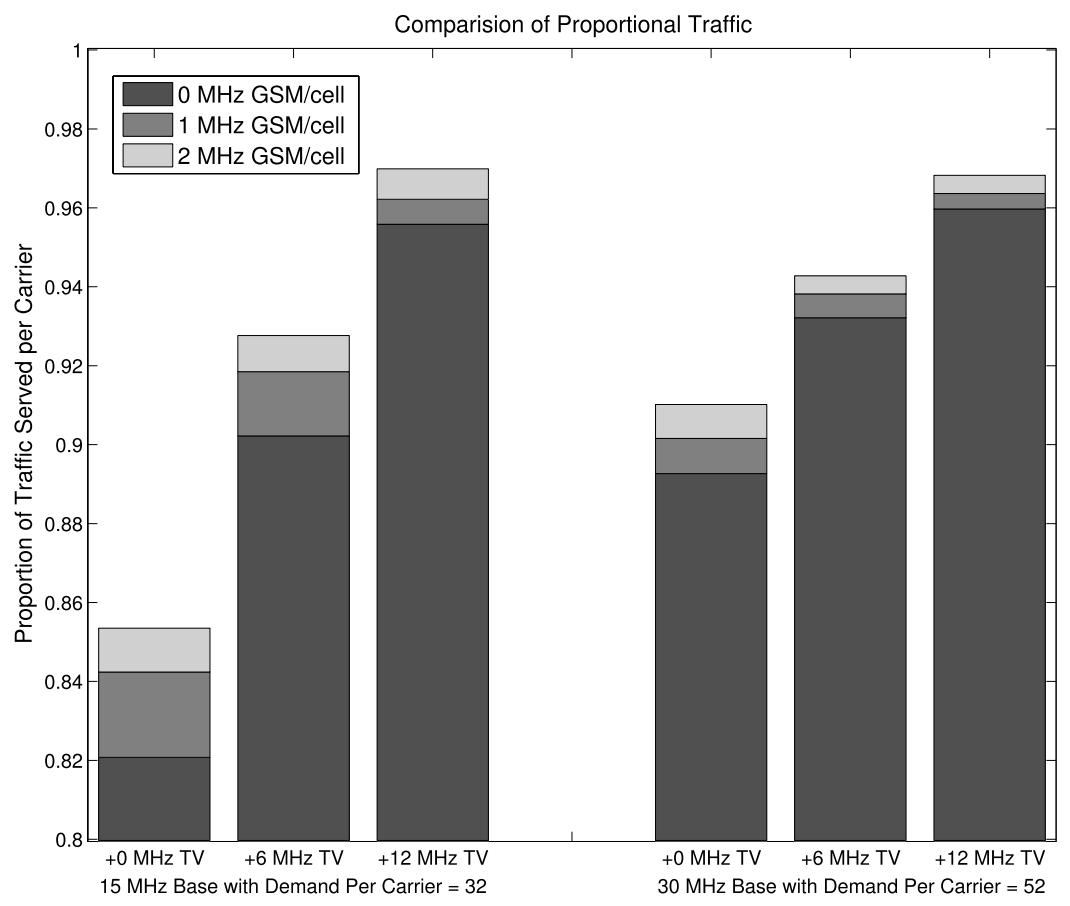

Fig. 19. Comparison of proportional traffic served given additional DSA spectrum for a $15 \mathrm{MHz}$ baseline with average demand of 32 channels per carrier (left bars) and $30 \mathrm{MHz}$ baseline with average demand of 52 channels per carrier (right bars).

proportional more traffic is served than the $12 \mathrm{MHz}$ case. The bars for the $30 \mathrm{MHz}$ case also show similar trends.
Thus, our experiments show that additional DSA spectrum results in slightly diminishing returns. 


\section{Conclusion and future work}

Our work has examined and proposed future methods by which DSA will be used in LTE+ wireless networks. In the first part of our paper, we examined the control planes and network elements affected by a DSA overlay. Through the SA framework, we examined two sets of operational procedures: service request supporting procedures and spectrum lease management procedures. Spectrum lease management of the SA framework can be used to monitor KPI and adjust spectrum leases for performance issues and policy changes. We illustrated spectrum lease management through different alarm and response procedures that could be dynamically used to adapt spectrum leases through the adjustment of spectrum access policies and rules. In the final section of the paper, through integer programs, we modeled the optimal assignment of DSA carriers to quantitatively illustrate the benefits of using a DSA overlay in LTE+ networks. In conclusion, this paper presented the operational effects of a DSA overlay in LTE+ networks through an SA framework. Defining and understanding these operational effects will be important for future LTE+ standards, infrastructure vendors, and network operators to deploy a DSA overlay in next generation wireless networks.

While studying the effects of DSA on next generation wireless networks, we have noted some potential open research areas, which we believe are of particular interest to network operators. The first area where we believe there is broad opportunity for research is specifically related to the cBS. There is the potential for additional research on determining the parameters of spectrum leases to be requested, based on load prediction through sequential and temporally relevant KPI. Using machine learning, is it possible for a cBS to predict future traffic patterns and spectral conditions to determine the most efficient spectrum leases? Which learning techniques will be useful in this regard? Spectrum sensing information could also play an important role in determining when to allow a spectrum lease request.

Additionally, we considered our integer programs as a snapshot in time, for specific assignment. During periodic intervals (e.g. half-hour intervals), this seems to be realistic for managing contention between network operators; however, opportunistic use of GSM spectrum could require a specialized sensing protocol to detect open time slots in GSM channels for opportunistic use, similar to [27]. Furthermore, instead of using integer programs to manage leases, sensing may be required to control shared use of opportunistic spectrum use between competitive operators. We believe there is a myriad of issues regarding the formulation of spectrum leases.

Our second proposed research area of research is to investigate the tradeoffs between spectrum policy paradigms. Specifically, we would like to compare scenarios similar to ones investigated in Section 5 with multiple competitive operators accessing DSA spectrum. In current regulation, secondary spectrum users must accept interference from one another, sharing the spectrum. We are interested in the effects of this spectrum policy and its effects on performance among multiple competitive operators. If competitive operators are aware of the spectrum conditions and greedily attempt to access the spectrum, how would this behavior manifest itself? How does this compare to the optimal? If competitive operators use an SAS, what methods would be useful to coordinate spectrum leases between secondary competitive operators? From examining these scenarios, we believe that we can provide useful feedback to regulators in developing future regulations for DSA.

\section{References}

[1] Global Mobile Suppliers Association, Evolution to LTE Report, GSM/3G Market/Technology Update, 2011.

[2] Federal Communications Commission, The benefits of additional spectrum, U OBI Technical Paper, 2010.

[3] R. Engelman, K. Abrokwah, G. Dillon, Report of the spectrum efficiency working group, Fed. Commun. Commission Spectr. Policy Task Force, 2002

[4] S. Parkvall, E. Dahlman, A. Furuskar, Y. Jading, M. Olsson, S. Wanstedt, K. Zangi, LTE-advanced-evolving LTE towards IMT-advanced, in: IEEE 68th Vehicular Technology Conference, VTC, 2008.

[5] W. Lehr, J. Chapin, Hybrid wireless broadband, in: 37th Research Conference on Communication, Information and Internet Policy, TPRC, 2009.

[6] L. Doyle, L. DaSilva, B. Ozgul, T. Forde, A letter from the editors, in: Physical Communications Special Issue on "Cognitive Radio for LTE Advanced and Beyond", 2011.

[7] Federal Communications Commission, In the Matter of: Unlicensed Operation in the TV Broadcast Bands (ET Docket No. 04-186) and Additional Spectrum for Unlicensed Devices Below $900 \mathrm{MHz}$ and in the $3 \mathrm{GHz}$ Band (ET Docket No. 02-380), FCC 10-174: Second Memorandum Opinion and Order, 2010.

[8] Ofcom, Implementing geolocation, consultation, 2010.

[9] Informa Telecoms and Media, Global market shares, 4G Americas Statistics, 2011. http://www.4gamericas.org/.

[10] M. Buddhikot, K. Ryan, Spectrum management in coordinated dynamic spectrum access based cellular networks, in: IEEE First International Symposium on New Frontiers in Dynamic Spectrum Access Networks, DySPAN, 2005

[11] M. Buddhikot, P. Kolodzy, S. Miller, K. Ryan, J. Evans, DIMSUMNet: new directions in wireless networking using coordinated dynamic spectrum access, in: Proceedings of the Sixth IEEE International Symposium on World of Wireless Mobile and Multimedia Networks, WOWMOM, 2005.

[12] V. Brik, E. Rozner, S. Banerjee, P. Bahl, DSAP: a protocol for coordinated spectrum access, in: IEEE First International Symposium on New Frontiers in Dynamic Spectrum Access Networks, DySPAN 2005.

[13] T. Baykas, J. Wang, M. Rahman, H. Tran, C. Song, S. Filin, Y. Alemseged, C. Sun, G. Villardi, C. Sum, et al. Overview of TV white spaces: current regulations, standards and coexistence between secondary users, in: IEEE 21st International Symposium on Personal, Indoor and Mobile Radio Communications Workshops, PIMRC, 2010.

[14] C. Stevenson, G. Chouinard, Z. Lei, W. Hu, S. Shellhammer, W. Caldwell, IEEE 802.22: the first cognitive radio wireless regional area network standard, IEEE Commun. Mag. (2009).

[15] J. Deaton, R. Irwin, L. DaSilva, The effects of a dynamic spectrum access overlay in LTE-advanced networks, in: IEEE 6th Internationa Symposium on New Frontiers in Dynamic Spectrum Access Networks, DySPAN, 2011.

[16] J. Peha, Spectrum management policy options, IEEE Commun. Surv. Tutor. (1998)

[17] T. Yucek, H. Arslan, A survey of spectrum sensing algorithms for cognitive radio applications, IEEE Commun. Surv. Tutor. (2009).

[18] 3rd Generation Partnership Project, TS 36.300 V10.3.0, Evolved Universal Terrestrial Radio Access (E-UTRA) and Evolved Universal Terrestrial Radio Access Network (E-UTRAN) Overall Description Release 10, 2011

[19] J.D. Deaton, C. Wernz, L.A. DaSilva, Decision analysis of dynamic spectrum access rules, in: IEEE Conference on Global Communication, GLOBECOM, 2011

[20] N. Theis, R. Thomas, L. DaSilva, Rendezvous for cognitive radios, IEEE Trans. Mobile Comput. (2010).

[21] P. Leaves, S. Ghaheri-Niri, R. Tafazolli, J. Huschke, Dynamic spectrum allocation in hybrid networks with imperfect load prediction, in: Third International Conference on 3G Mobile Communication Technologies, 2002 
[22] Rysavy Research, Mobile broadband capacity constraints and the need for optimization, 2009

[23] R. Kwan, C. Leung, A survey of scheduling and interference mitigation in LTE, Hindawi J. Electr. Comput. Eng. (2010).

[24] IBM, ILOG CPLEX Optimizer, 2011.

http://www-01.ibm.com/software/integration/optimization/cplexoptimizer/.

[25] Federal Communications Commission, 10-198: In the Matter of: Promoting More Efficient Use of Spectrum Through Dynamic Spectrum Use Technologies (ET Docket No. 10-237), FCC 10-198: Notice of Inquiry, 2010.

[26] M. Wellens, P. Mähönen, Lessons learned from an extensive spectrum occupancy measurement campaign and a stochastic duty cycle model, Springer Mobile Netw. Appl. (2010).

[27] V. Blaschke, C. Kloeck, J. Weiss, T. Renk, F. Jondral, Opportunistic WiMAX-GSM coexistence, IET Commun. (2008).

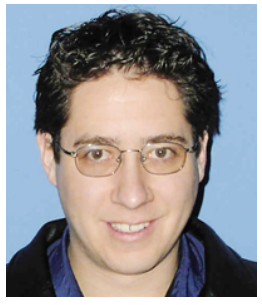

Juan D. Deaton is currently employed as a Cellular Systems Engineer at the Idaho National Lab and a Ph.D. Student at Virginia Tech. Before becoming a graduate student, Juan's work involved researching options for wireless airborne emergency communications and vulnerabilities of VolP applications. At Virginia Tech, his focus is in wireless cognitive networking under the advisement of Dr. Luiz DaSilva.

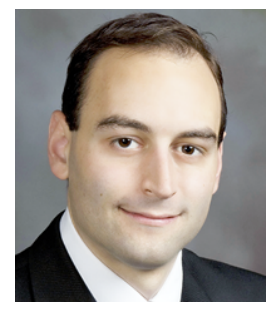

Ryan E. Irwin has been a graduate student at Virginia Tech since the fall of 2007. Within the ECE department he is part of the Wireless @ Virginia Tech research group. His focus is in wireless networking and communication under the co-advisement of Dr. Allen MacKenzie and Dr. Luiz DaSilva. Ryan obtained a B.S. in computer engineering from Mississippi State University in May of 2007.

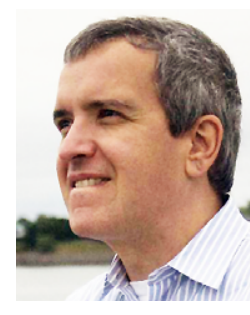

Luiz A. DaSilva is currently on leave from Virginia Tech's Bradley Department of Electrical and Computer Engineering, where he has been a faculty member since 1998 . He currently holds the Stokes Professorship in Telecommunications in the Department of Electronic and Electrical Engineering at Trinity College Dublin, in Ireland. Prof. DaSilva's research focuses on distributed and adaptive resource management in wireless networks, and in particular cognitive radio networks and the application of game theory to wireless networks. He is currently a principal investigator on research projects funded by the National Science Foundation, DARPA, and the European Commission under Framework Programme 7. Prof. DaSilva is a Senior Member of IEEE and a member of the ASEE and of ACM. In 2006, he was named a College of Engineering Faculty Fellow at Virginia Tech. 